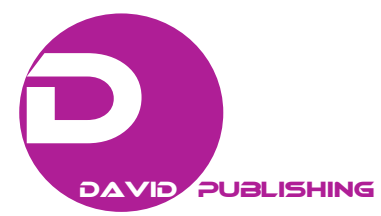

\title{
Strangulated Obturator Hernia: Difficult Diagnosis
}

\author{
Hamri Asma, Narjis Youssef and Benelkhaiat Ridouan \\ Department of Surgery, Mohammed the Sixth University Hospital, Marrakech 40000, Morocco
}

\begin{abstract}
The obturator hernia is rare and its diagnosis is frequently missed. It is a rare cause of intestinal obstruction. Patients present with few clinical signs to identify the cause, apart from symptoms of intestinal obstruction. Therefore, obturator hernias are often diagnosed at laparotomy with a high motality rate. CASE REPORT: We recently encountered this lesion in a 75 year old woman presented with a history of right iliac fossa pain, bilious vomiting, abdominal distension. Non-specific findings on examination and blood tests made the diagnosis difficult, however, a CT scan of her abdomen revealed the site of the obstruction and the patient was taken to theatre for emergency surgery. She underwent a midline laparotomy and resection and anastomosis of small bowel and repair of the strangulated right obturator hernia. She presented a tachyrdia six days after surgery. The patient died of peritonitis postoperative (anastomotic dehiscence). The high mortality rate associated with this type of abdominal hernias requires a high index of suspicion to facilitate rapid diagnosis and prompt surgical intervention if the survival rate is to be improved.
\end{abstract}

Key words: Obturator hernia, laparotomy, intestinal obstruction, strangulation.

\section{Background}

Strangulated obturator hernia is rare, but has a mortality of $10 \%$ to $20 \%$ (Hershman, Reilly and Swift 1986), partly because the diagnosis is made in only about $30 \%$ of patients before laparotomy [1].

The real incidence of obturator hernias is unknown. They are thought to constitute fewer than $1 \%$ of all hernias worldwide. Bjork and colleagues reported the incidence at about $0.073 \%$ of all hernias repaired at the Mayo Clinic [2]. Obturator hernia was initially described by Arnaud de Ronsil in 1724 and was first successfully repaired in 1851 by Henry Obre. Females are affected 6 times more frequently than males. A thin body habitus is a strong risk factor.

\section{Case Report}

We recently encountered this lesion in a 75 year old woman presented with a history of right iliac fossa pain, bilious vomiting, abdominal distension. Non-specific findings on examination and blood tests made the diagnosis difficult, however, a CT scan of her abdomen revealed the site of the obstruction and the patient was

Corresponding author: Benelkhaiat Ridouan, Ph.D., research field: surgery. taken to theatre for emergency surgery. She underwent a midline laparotomy and resection and anastomosis of small bowel and repair of the strangulated right obturator hernia. She presented a tachyrdia six days after surgery. The patient died of peritonitis postoperative (anastomotic dehiscence).

\section{Discussion}

An obturator hernia is an extremely uncommon pelvic and intra-abdominal hernia. It is a rare condition first described in 1724 by Arnaud de Ronsil at the Royal Academy of Sciences in Paris [3]. The Obturator hernia has a right to left ratio of 3:1. Bilateral obturator hernias though rare have been reported. There is a female pre-ponderance because of their wider pelvis and parity which causes the pelvic peritoneum to go lax. Other predisposing. The obturator canal is an opening in the superolateral part of the obturator foramen. It contains the obturator nerve and vessels. The canal is $2-3 \mathrm{~cm}$ long and $1 \mathrm{~cm}$ wide, and it is usually filled with fat, allowing no space for a hernia [4]. The fat disappears in patients who have massive body weight loss or are very thin. Women are affected more often because they have a broader pelvis and larger obturator canal. Multiple pregnancies may also be a contributing 
factor, although there is still controversy that this is a contributory factor. The clinical features are thigh or knee pain in the affected side, recurrent bouts of intestinal obstruction with cramping abdominal pain, nausea, and vomiting. the diagnosis of obturator hernia is difficult. Sometimes, the hernia may be felt as a tender swelling in the region of the obturator foramen on vaginal or rectal examination. HowshipRomberg sign, which refers to ipsilateral groin pain radiating down the thigh as a result of irritation of the obturator nerve, is a characteristic sign of obturator hernia. The Hannington-Kiff sign is considered more specific than Howship-Romberg sign in the diagnosis of obturator hernia. Plain radiographs often show nonspecific findings of small bowel obstruction and are seldom helpful in diagnosing obturator hernia. Noting a gas shadow in the obturator foramen area may be helpful. A barium enema or small bowel series can be useful if a bowel loop is in the obturator canal, but a barium study is more time consuming in diagnosing a case of acute abdomen. Ultrasonography is useful and reliable in the diagnosis of obturator hernia, but it is often limited by the relative inaccessibility of this deep region and is operator dependent. Surgery through midline incision is preferred for an obturator hernia, because it gives the best exposure, allows reduction of hernia content, and facilitates bowel resection if necessary [5]. Resection of the involved portion of the bowel is sometimes required because of gangrenous changes or perforation.
The mortality rate for patients with an obturator hernia is still high. Reported mortality rates ranged from $12 \%$ to $70 \%$. The high mortality is often related to delayed diagnosis, treatment, and the debilitated physical condition of the patients [6].

\section{Conclusion}

The obturator hernia is an important diagnosis to be considered in elderly patients with intestinal obstruction. Early diagnosis and surgical treatment contribute significantly to reduce the mortality and morbidity rates.

\section{References}

[1] Gray, S. W., Skandalakis, J. E., Soria, R. E., and Rowe Jr., J. S. 1974. "Strangulated Obturator Hernia." Surg. 75 (1): 20-7.

[2] Bjork, K. J., Mucha, P. Jr., and Cahill, D. R. 1988. "Obturator Hernia." Surg. Gynecol. Obstet. 167 (3): 217-22.

[3] Lo, C. Y., Lorentz, T. G., and Lau, P. W. 1994. "Obturator Hernia Presenting as Small Bowel Obstruction." American Journal of Surgery 167 (4): 396-8.

[4] Kulkarni, R. S., Punamiya, R. A., Naniwadekar, G. R., Janugade, H. B., Chotai, T. D., Singh, T. V., and Natchair, A. 2013. "Obturator Hernia: A Diagnostic Challenge." International Journal of Surgery Case Reports 4 (7): 606-8.

[5] Pandey, R., Maqbool, A., and Jayachandran, N. 2009. "Obturator Hernia: A Diagnostic Challenge." Hernia 13 (1): 97-9.

[6] Shipkov, C. D., Uchikov, A. P, and Grigoriadis, E. 2004. "The Obturator Hernia: Difficult to Diagnose, Easy to Repair." Hernia 8 (2): 155-7. 